\title{
A influência da osteoartrite de joelho no equilíbrio e marcha de indivíduos idosos
}

\author{
The influence of knee osteoarthritis on the balance and gait of elderly individuals \\ La influencia de la osteoartritis de rodilla en el equilibrio y la marcha de los ancianos
}

\author{
Marianna de Melo Salemi \\ ORCID: https://orcid.org/0000-0001-5804-6181 \\ Universidade Federal de Pernambuco, Brasil \\ E-mail: mariannasalemifisio@gmail.com \\ Vanessa Maria da Silva Alves Gomes \\ ORCID: https://orcid.org/0000-0002-6866-6808 \\ Universidade Federal de Pernambuco, Brasil \\ E-mail: vanessaalvesfta@gmail.com \\ Juliana Alves do Monte \\ ORCID: https://orcid.org/0000-0003-2968-5763 \\ Universidade Católica de Pernambuco, Brasil \\ E-mail: julianamontefisio@gmail.com \\ Augusto Cesar Bezerra Lopes \\ ORCID: https://orcid.org/0000-0002-3566-0055 \\ Faculdade Facottur, Brasil \\ E-mail: augustocesarb185@gmail.com \\ Eudson José Santos do Monte \\ ORCID: https://orcid.org/0000-0002-1871-6619 \\ Faculdade Facottur, Brasil \\ E-mail: eudson41@gmail.com \\ Harrison Euller Vasconcelos Queiroz \\ ORCID: https://orcid.org/0000-0001-7906-2981 \\ Faculdade Facottur, Brasil \\ E-mail: harrisoneuller@gmail.com \\ Valéria Conceição Passos de Carvalho \\ ORCID: https://orcid.org/0000-0001-8314-9000 \\ Universidade Católica de Pernambuco, Brasil \\ E-mail: valeriapassos@gmail.com \\ Marina de Lima Neves Barros \\ ORCID: https://orcid.org/0000-0002-3544-0538 \\ Universidade Católica de Pernambuco, Brasil \\ E-mail: marinalnbarros@gmail.com \\ Érica Patrícia Borba Lira Uchôa \\ ORCID: https://orcid.org/0000-0003-4099-1876 \\ Universidade Católica de Pernambuco, Brasil \\ E-mail: ericaluchoa@gmail.com
}

\begin{abstract}
Resumo
Objetivo: analisar a influência que a osteoartrose tem sobre o equilíbrio e a marcha de pacientes idosos. Metodologia: Trata-se de um estudo do tipo observacional em idosos de ambos os sexos com osteoartrite de joelho diagnosticada. Inicialmente foi realizado uma palestra sobre os objetivos do estudo e após assinatura do Termo de Consentimento Livre e Esclarecido foi realizado a coleta de dados sociodemográfico; avaliação da dor pela Escala visual analógia; avaliação do equilíbrio pela Escala de Equilíbrio de Berg, avaliação da mobilidade e marcha através do Time Up and Go e Teste de Tinetti. Resultados: Foram selecionados para o estudo 11 idosos com osteoartrose, todos do sexo feminino, com média de idade de 75,6 anos. Houve correlação entre o aumento da idade com a piora na mobilidade e equilíbrio dos idosos. Considerações finais: Concluiu-se nesta pesquisa, que o envelhecimento é um processo fisiológico e progressivo, no qual vários sistemas do organismo são comprometidos, principalmente o sistema musculoesquelético. Os aspectos como o equilíbrio e morbidade tem uma forte relação com o avanço da idade, podendo ter comprometimento físico e psicológico.
\end{abstract}

Palavras-chave: Equilíbrio; Idosos; Marcha; Osteoartrose.

\begin{abstract}
Aim: To affect the influence that osteoarthrosis has on the balance and gait of elderly patients. Method: This is an observational study in elderly people of both genders with diagnosed knee osteoarthritis. Initially, a lecture was held on the objectives of the study and after signing the Informed Consent Term, the collection of sociodemographic data was carried out; pain assessment using the visual analogue scale; balance assessment using the Berg Balance Scale, mobility and gait assessment using the Time
\end{abstract}


Up and Go and Tinetti Test. Results: Eleven elderly people with osteoarthritis, all female, with a mean age of 75.6 years, were selected for the study. There was a correlation between increasing age and worsening mobility and balance in the elderly. Conclusion: It was concluded in this research, that aging is a physiological and progressive process, in which several body systems are compromised, mainly the musculoskeletal system. Aspects such as balance and morbidity have a strong relationship with advancing age and may have physical and psychological impairment.

Keywords: Balance; Elderly; Gait; Osteoarthritis.

\section{Resumen}

Objetivo: analizar la influencia que tiene la osteoartrosis en el equilibrio y la marcha de los pacientes ancianos. Metodología: Se trata de un estudio observacional en personas mayores de ambos sexos con osteoartritis de rodilla diagnosticada. Inicialmente se realizó una charla sobre los objetivos del estudio y luego de la firma del Término de Consentimiento Informado se llevó a cabo la recolección de datos sociodemográficos; evaluación del dolor mediante la escala analógica visual; evaluación del equilibrio mediante la escala de equilibrio de Berg, evaluación de la movilidad y la marcha mediante el test Time Up and Go y Tinetti. Resultados: Se seleccionaron para el estudio 11 ancianos con artrosis, todas mujeres, con una edad media de 75,6 años. Hubo una correlación entre el aumento de la edad y el empeoramiento de la movilidad y el equilibrio en los ancianos. Consideraciones finales: En esta investigación se concluyó que el envejecimiento es un proceso fisiológico y progresivo, en el que se ven comprometidos varios sistemas corporales, principalmente el sistema musculoesquelético. Aspectos como el equilibrio y la morbilidad tienen una fuerte relación con la edad avanzada y pueden tener un deterioro físico y psicológico.

Palabras clave: Equilibrio; Ancianos; Marcha; Osteoartrosis.

\section{Introdução}

O envelhecimento é um processo fisiológico, que pode ser considerado como um conjunto de alterações estruturais e funcionais desfavoráveis do organismo que se acumulam de forma progressiva, especificamente em função do avanço da idade. Quando se tem uma idade mais avançada todos os sistemas do nosso corpo se tornam mais fragilizados, tendo alterações em suas estruturas (Monte et al., 2020; Zis et al., 2017).

Essas modificações geralmente vêm acompanhadas pelas dores crônicas, na qual estão relacionadas ao sistema musculoesquelético e prejudicam o desempenho de habilidades motoras, dificultando a adaptação do indivíduo ao meio ambiente, desencadeando modificações psicológicas e sociais Fatores como depressão, dependência, afastamento social, mudanças na sexualidade, alterações na dinâmica familiar, desequilíbrio, marcha alterada, desesperança, sentimento de morte e outros, encontram-se associados a quadros de dor crônica. A dor passa a ser o centro, direciona e limita as decisões e comportamentos do indivíduo. A impossibilidade de controlá-la traz sempre sofrimento físico e psíquico e desta forma, todos esses fatores associados parecem aumentar a morbidade entre os idosos e onerar o sistema de saúde. (Diegelmann, Schilling \& Wahl, 2016; Rathbun et al., 2018).

A osteoartrose é uma típica patologia que está associada ao avanço da idade (Elwyn et al., 2017). É resultante de um processo degradativo cartilaginoso complexo e multifatorial (Suzuki et al., 2019). Estudos comprovam que a incidência de osteoartrose é maior no sexo feminino e que as articulações mais frequentemente acometidas são as mãos, os pés, os joelhos, a coluna (lombar e cervical) e os quadris (Dulay, Cooper \& Dennison, 2015; Peeler et., 2015; Rodriguez-Merchan \& De La Corte-Rodriguez, 2019; Suzuki et al., 2019).

$\mathrm{Na}$ osteoartrose, quer primária ou secundária, a cartilagem é o tecido que mais sofre com as alterações, a qual perde sua natureza homogênea, é rompida e fragmentada, com fibrilação, fissuras e ulcerações. Com o avanço da patologia, a cartilagem passa a não existir e áreas de osso subcondral ficam expostas (Hussain et., 2016).

Por sua vez, devido a todas essas alterações patológicas, a dor crônica está como um dos principais sintomas da doença, acarretando desta forma inúmeras consequências ao indivíduo. Tais consequências são especificas da osteoartrose, pois é provável que devido ao nível de dor que o paciente refere, a marcha e o equilíbrio do indivíduo sejam afetados. (Peeler et al., 2015)

Pesquisas revelam que pacientes com osteoartrose, apresentam dor que aparece ou piora com o início dos movimentos, rigidez matinal de curta duração, crepitação, diminuição da função articular, além da hipotrofia muscular. Quando a 
osteoartrose se localiza no joelho, as chances do surgimento de um quadro incapacitante são maiores, podendo restringir a marcha, coordenação, equilíbrio, subir escadas, levantar entre outros gestos de atividades da vida diária, aumentando desta forma a dependência funcional do indivíduo. (Hussain et al., 2016; Kan et al., 2019).

Diante do exposto, o objetivo deste trabalho foi analisar a influência que a osteoartrose tem sobre o equilíbrio e a marcha de pacientes idosos.

\section{Metodologia}

Trata-se de um estudo do tipo observacional, descritivo, de corte transversal e abordagem quantitativa. Segundo Pereira et al. (2018) na abordagem quantitativa faz-se uma coleta de dados numéricos com medições de grandezas, gerando um conjunto de dados que são analisados por meio de técnicas matemáticas. O presente estudo está vinculado ao projeto de pesquisa "Recursos de Avaliação e Intervenção Fisioterapêuticos em Alterações Osteomioarticulares na Saúde de Indivíduos Adultos e Idosos", com o número de CAAE: 09962313.8.0000.5206, foi aprovado pelo comitê de ética e pesquisa em seres humanos da Universidade Católica de Pernambuco, com o número de parecer: 226.764, atendendo assim à resolução 466/12 do Conselho Nacional de Saúde. O estudo foi realizado nos laboratórios especializados em Fisioterapia e Terapia Ocupacional de uma clínica escola da cidade do Recife, estado de Pernambuco.

A amostra foi composta por 11 idosos de ambos os sexos, de forma intencional, cujo recrutamento foi realizado através da lista de espera de idosos da Clínica Escola de Fisioterapia. Como critérios de inclusão foram adotados: Idosos de ambos os sexos, com idade de 60 anos ou mais e diagnóstico médico de osteoartrose de joelho. Os critérios de exclusão foram: pacientes que tenham comprometimento cognitivo, que os impeça de responder os questionários, apresentem perdas de visão, audição e outras doenças associadas que comprometam a qualidade de vida, associação com patologias prévias como: amputações, sequelas de traumas e presença de deformidades.

Inicialmente, foi realizada uma palestra explicativa expondo aos pacientes quais foram os pontos avaliados, como foi feita a coleta de informações e apresentando os benefícios dos resultados na qualidade de saúde do idoso. E, aqueles que aceitaram participar da pesquisa assinaram o Termo de consentimento livre e esclarecido (TCLE), dando seu consentimento a participação no estudo.

Após assinatura do TCLE, foi aplicado o questionário sociodemográfico e clínico, a fim de traçar um perfil de dados dos idosos, apresentando dados como sexo, idade, altura, peso, uso de cigarro, medicação e álcool (quantidade e frequência), realização de atividade física. Em seguida, foi aplicada a Escala Visual Analógica (EVA), a qual consiste em auxiliar na aferição da intensidade da dor do paciente. Trata-se de uma régua de $10 \mathrm{~cm}$ onde o extremo 0 (zero) é a ausência total de dor e o extremo 10 (dez) o nível de dor máxima. (Campbell \& Lewis, 1990).

Dando continuidade a investigação, os pacientes foram submetidos a testes e questionários, no qual demonstraram resultados relacionados ao equilíbrio e a marcha que esses pacientes evidenciam devido a osteoartrose de joelho.

Para análise do equilíbrio, foi utilizada a Escala de Equilíbrio de Berg (EEB) na qual é constituída por 14 tarefas que envolvem o equilíbrio estático e dinâmico tais como alcançar, girar, transferir-se, permanecer em pé e levantar-se, onde cada item possui 5 alternativas (de 0 a 4 pontos). A realização das tarefas é avaliada através de observação e a pontuação pode variar de 0 a 56 pontos, onde 0 representa um déficit grave de equilíbrio e 56 um equilíbrio excelente (Ferreira et al., 2019; Miyamoto et al., 2004).

O Timed Up and Go Test (TUG) é um teste simples e rápido para avaliar a mobilidade e habilidade funcional dos idosos durante a marcha, capaz de detectar os problemas de equilíbrio que afetam as capacidades de mobilidade diária dos pacientes idosos. A partir disso pode-se concluir que pacientes que levam menos de 10 segundos para realizar a tarefa são considerados 
capazes e independentes na realização das Atividades de Vida Diária (AVDs). Acima de 30 segundos, os indivíduos são considerados dependentes na maioria das AVDs e nas capacidades de mobilidade. Os resultados da escala variam com o nível de fraqueza muscular e espasticidade (Dutra, Cabral \& Carvalho, 2016).

O Teste de Tinetti, classifica aspectos da marcha como a velocidade, a distância do passo, a simetria e o equilíbrio em pé, o girar e também as mudanças com os olhos fechados. É uma escala confiável para detectar mudanças significativas durante a marcha e mudanças de posição. A utilização deste instrumento de avaliação tem importantes implicações na qualidade de vida dos idosos, uma vez que possibilita ações preventivas, assistenciais e de reabilitação (Nogueira et al., 2017; Gomes, 2003).

Após a avaliação, foi elaborada uma planilha de resultados no Microsoft Excel® 2007, onde foram apresentadas as estatísticas descritivas por meio de distribuição de frequências para as variáveis categóricas e médias e medianas com suas respectivas variações para as variáveis quantitativas. Na análise das escalas as comparações foram feitas utilizando o valor da mediana por não ter sido comprovada uma distribuição normal dos dados. O teste de normalidade foi realizado aplicando o teste de Komogorov-Smirnov. O teste utilizado na comparação das medianas dos escores segundo a condição de atividade física foi o Mann-Whitney. Na análise da correlação das escalas com a idade, o coeficiente de correlação de Spearman foi aplicado. A significância estatística adotada no estudo foi de 5\% (p < 0,05). O software utilizado na análise foi o STATA versão 12.0 .

\section{Resultados}

Foram selecionados para o estudo 11 idosos com osteoartrose, todos do sexo feminino, com média de idade de 75,6 anos, idade mínima de 60 e máxima de 87 anos. O índice de massa corpóreo médio dos pacientes foi de $29,4 \mathrm{~kg} / \mathrm{m}^{2}$ (em média, pacientes sobrepesos). O uso de medicação foi referido por $81,8 \%$ dos pesquisados. Em relação a hábitos, nenhum dos idosos selecionados faziam uso de bebida alcoólica, 2 eram tabagistas (18,2\%) e 5 deles faziam atividade física regular (Tabela 1).

Tabela 1. Perfil dos pacientes idosos portadores de osteoartrose de joelho

\begin{tabular}{ll}
\hline Características & Estatística \\
\hline Idade $(\mathrm{em} \text { anos })^{\mathrm{a}}$ & $75,6 \pm 8,4(60 ; 87)$ \\
IMC $\left(\mathrm{kg} / \mathrm{m}^{2}\right)^{\mathrm{a}}$ & $29,4 \pm 4,5(21,3 ; 36,1)$ \\
Uso de medicação & \\
Sim & $9(81,8 \%)$ \\
Não & $2(18,2 \%)$ \\
Tabagista & \\
Sim & $2(18,2 \%)$ \\
Não & $9(81,8 \%)$ \\
Atividade física & \\
Sim & $5(45,4 \%)$ \\
Não & $6(54,6 \%)$ \\
\hline
\end{tabular}


Segundo escala EVA de dor, a média do escore referidos pelos pacientes foi de $5 \pm 2$ pontos, com escore mínimo de 2 e máximo de 8 pontos, mais da metade dos pacientes referiram nível de 6 ou mais pontos quanto a intensidade da dor (Figura $1)$.

Figura 1. Medidas da escala de dor dos pacientes idosos portadores de osteoartrose de joelho.
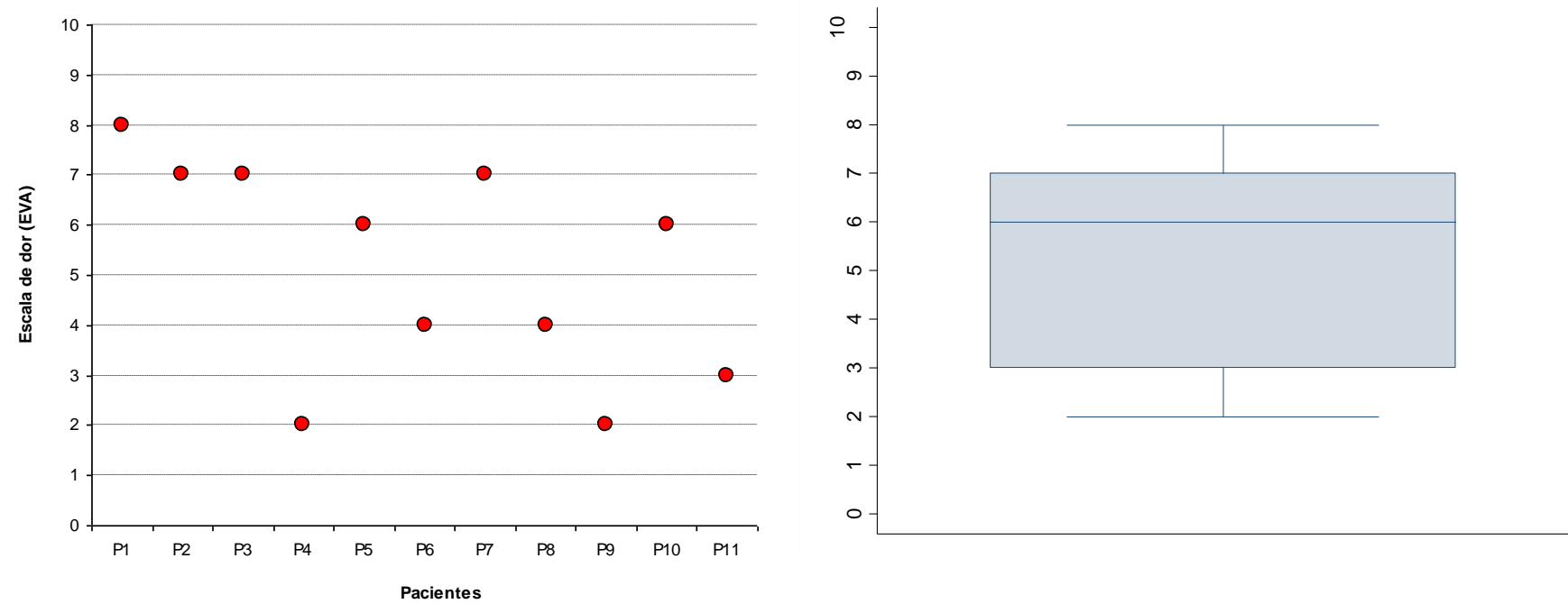

Fonte: Pesquisa Direta (2020).

Na avaliação do teste de Tinetti, a pontuação relativa ao equilíbrio apresentou uma maior média quando comparado ao da marcha, porém os valores medianos foram semelhantes. O total de pontos da escala de Tinetti Foi de 21,4 pontos, variando entre 14 e 28 pontos (Tabela 2).

Tabela 2. Avaliação do equilíbrio e marcha, medidos pela escala de Tinetti, dos pacientes idosos portadores de osteoartrose de joelho.

\begin{tabular}{ll}
\hline Escala de Tinetti & Pontuação \\
\hline Equilíbrio & $11,8 \pm 3,2 ; 11(8 ; 16)$ \\
Marcha & $9,6 \pm 2,8 ; 11(3 ; 12)$ \\
Total & $21,4 \pm 5,0 ; 20(14 ; 28)$ \\
\hline
\end{tabular}

Média \pm DP; mediana (mínimo; máximo); DP = Desvio Padrão.

Fonte: Pesquisa Direta (2020).

Na Figura 2 observa-se que na distribuição dos pontos segundo a escala de Berg, metade dos pacientes tem pontuação abaixo de 36, o que corresponde, segundo Shumway-Cook, et al, elevado risco de queda. A pontuação média dos idosos foi de aproximadamente 39 pontos, com mínimo de 26 e máximo de 51 pontos. 
Figura 2. Avaliação da escala de BERG balance dos pacientes idosos portadores de osteoartrose de joelho.

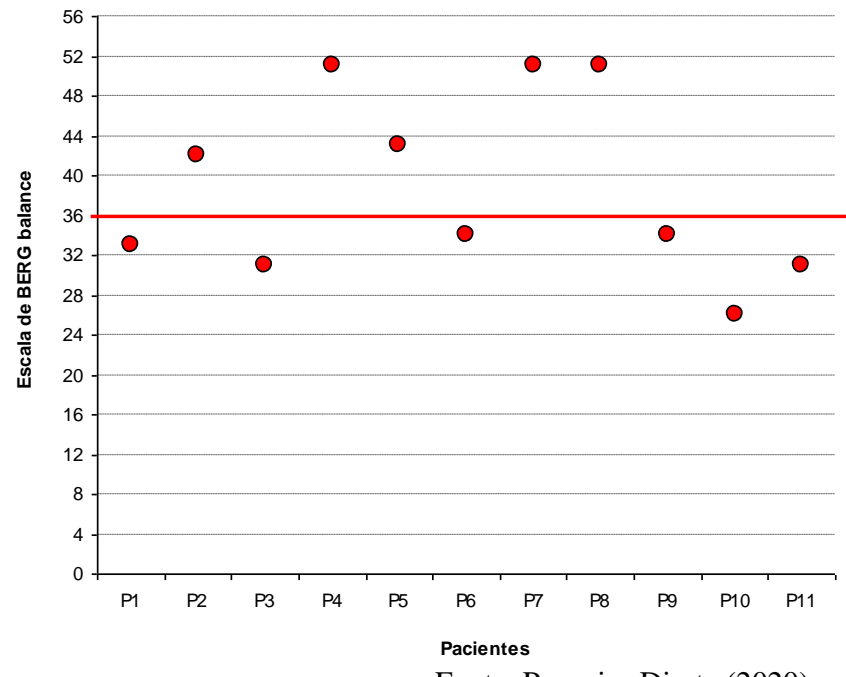

Ponto de corte sugerido por ShumwayCook, et al (2003), para elevado risco de queda.

Fonte: Pesquisa Direta (2020).

$\mathrm{Na}$ avaliação do teste time up go, dois pacientes apresentaram valores acima do esperado, um com tempo de $46 \mathrm{e}$ outro com tempo de 60 segundos, um com 87 anos de idade e o outro com 83 anos, respectivamente, ambos com alto risco de queda (26 e 34 pontos) e baixo escore de Tinetti (14 e 19 pontos). A média de tempo alcançado pelos idosos pesquisados foi de 9,8 segundos, onde $50 \%$ dos idosos fizeram as atividades em menos de 11 segundos.

Figura 3. Avaliação do tempo de realização do teste TUG dos pacientes idosos portadores de osteoartrose de joelho.
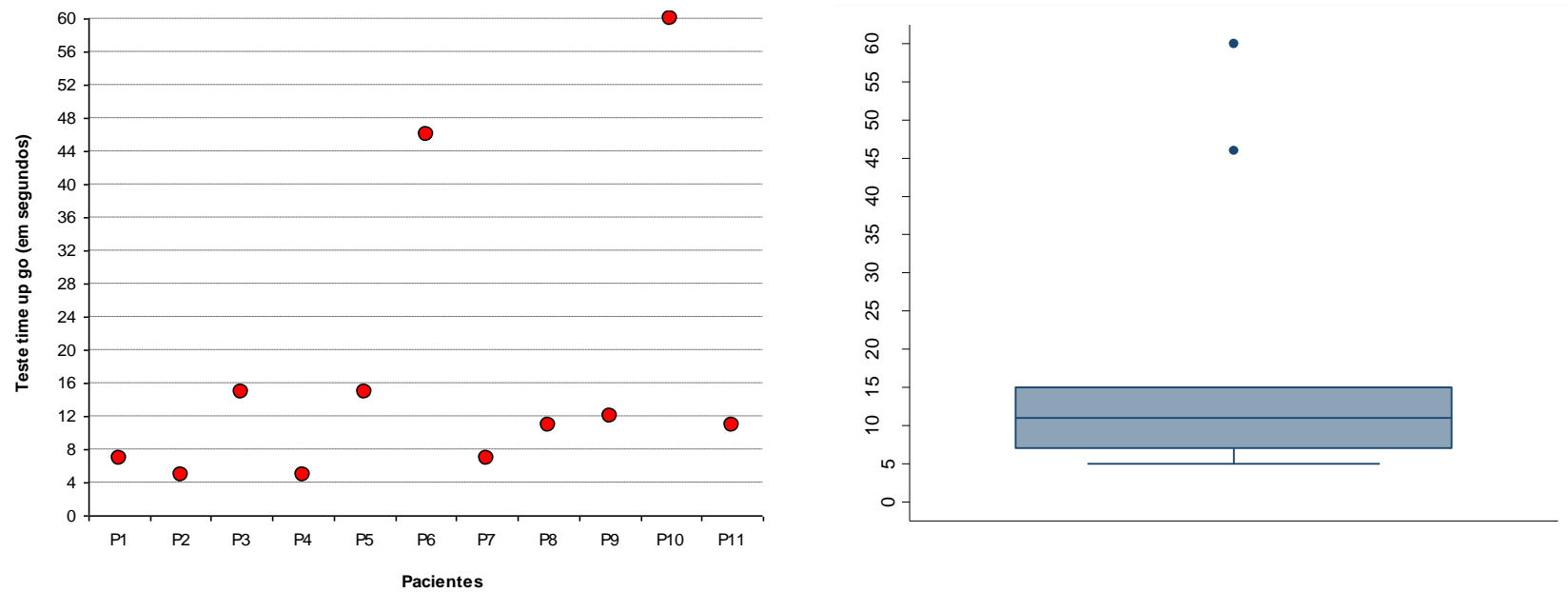

Média \pm DP: 9,8 \pm 4 segundos; Mediana; 11 segundos; Mínimo e Máximo: 5 a 60 segundos Fonte: Pesquisa Direta (2020)

Correlacionando os escores de dor, mobilidade e equilíbrio com a idade, observa-se que há significância estatística em relação a escala de Tinetti e escala de Berg, ambas com correlações fortes e negativas. Isso significa dizer que o aumento da idade está relacionado a uma piora na mobilidade dos idosos e na piora do equilíbrio. Em relação a dor e o tempo de realização de atividades a idade não se mostrou correlacionadas, o que sugere independência desses fatores quanto a idade do paciente. 
Figura 4. Correlação da escala de dor, escala de Tinetti, escala de BERG balance e TUG e a idade dos pacientes idosos portadores de osteoartrose de joelho.
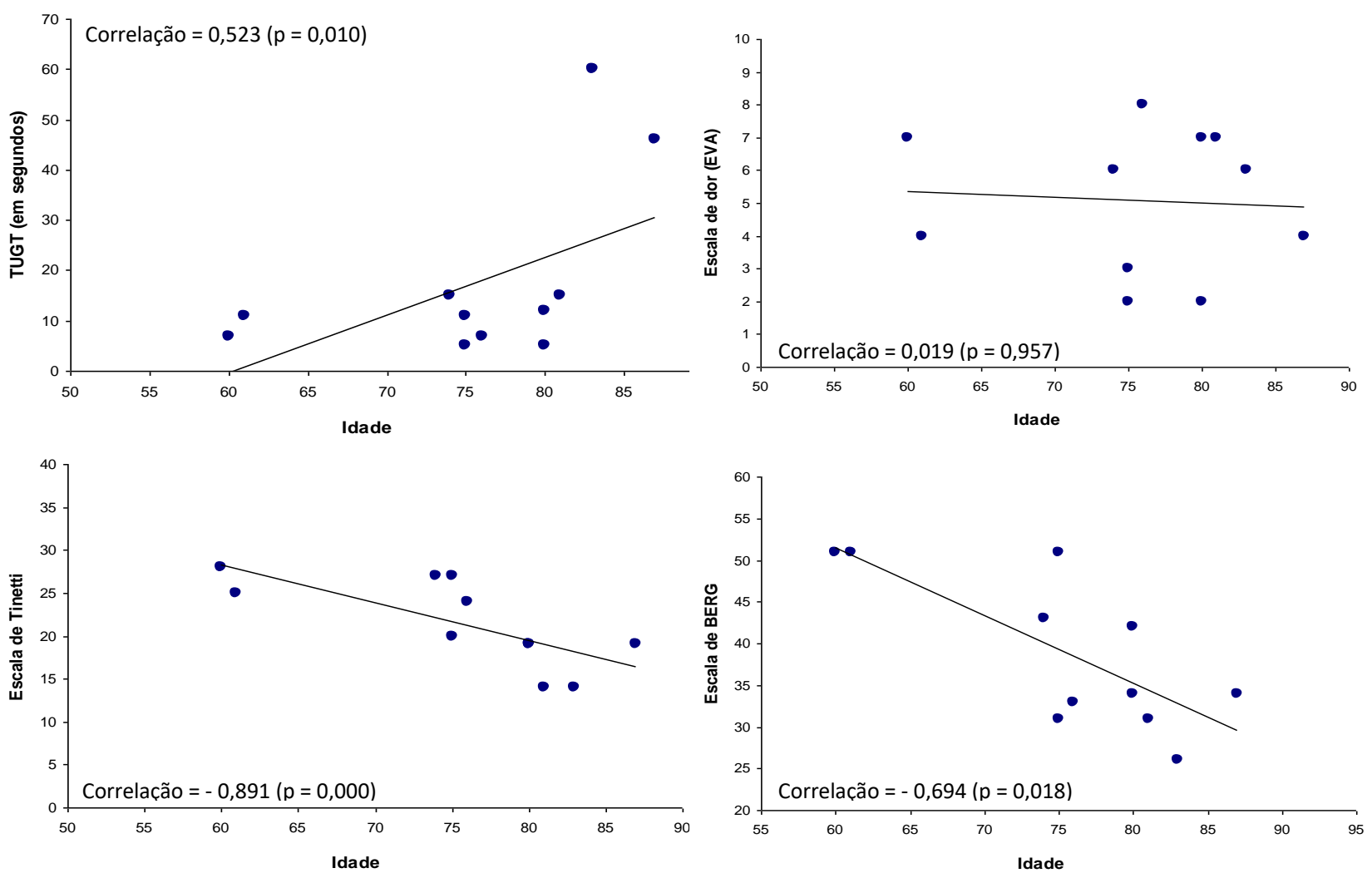

Fonte: Pesquisa Direta (2020).

\section{Discussão}

Dentre as variáveis sócioepidemiológicas foi possível evidenciar a presença apenas do sexo feminino entre os idosos incluídos no estudo. Corroborando com o estudo realizado por Antunes et al. (2020), com uma alta frequência de mulheres $(84,2 \%)$ entre os idosos com osteoartrite de joelho, assim como, no estudo de Santos et al. (2015), também com idosos com OA, em que a frequência de mulheres foi de $69,5 \%$, predominando o sexo feminino.

Em todo o mundo, as mulheres tendem a exibir uma mortalidade menor que aos homens, porém isso não significa que estas mulheres apresentem uma melhor qualidade de vida, pois há evidência de que as mulheres idosas suportam maiores condições de doenças crônicas, o que acarreta um declínio funcional mais acentuado. Um fator que pode influenciar na OA são os fatores hormonais nas mulheres, pois normalmente apresentam muitos anos de vida após a menopausa. No período pósmenopausa são comuns os desconfortos articulares causados por vários fatores, por exemplo, o excesso de peso, que acaba por sobrecarregar as estruturas musculoesqueléticas (Souza et al., 2017; Selbac et al., 2018).

Segundo o estudo de Hunter \& Bierna-Zeinstra (2019), a OA é uma condição clínica que afeta pessoas de diferentes faixas etárias, podendo surgir em indivíduos no início da fase adulta, porém no estudo de Franco et al. (2020), observou-se que as chances do surgimento da osteoartrite aumenta com a idade, em concordância com Yamada et al. (2018), no qual relatam que a OA é pouco comum em indivíduos abaixo dos 40 anos de idade e mais frequente após os 60 anos. Acredita-se que o aumento da incidência da patologia com a idade provavelmente seja consequência de várias mudanças biológicas como aumento da flacidez dos ligamentos ao redor das articulações e fraqueza da musculatura tornado as articulações mais instáveis ficando suscetíveis a lesões (Glyn-Jones et al., 2015). 
De acordo com Gomes-Neto et al. (2016), a obesidade representa um fator de risco somado a idade para o desenvolvimento da OA, além disso, o seu estudo demonstrou que os idosos obesos comparados aos não obesos, tiveram redução de desempenho no TUG, na velocidade da marcha rápida e no TC6, apresentaram maior intensidade de dor nos itens: “executar tarefas domésticas pesadas", “descer escadas", "curvar-se em direção ao chão" e "levantar-se da cama". Diante disso, acredita-se que o risco de ocorrência desta doença parece aumentar proporcionalmente ao IMC, como afirma o estudo de Pacca et al. (2018). Assim, como no presente estudo, as evidências sugerem que o sobrepeso é um importante fator de risco para a ocorrência de OA de joelho.

No presente estudo, foi avaliado o nível de dor que paciente referia, pela EVA e foi mensurada de forma estática, ou seja, no momento em que não estava realizando nenhum movimento, porém durante a avaliação alguns pacientes relataram piora da dor, quando realizava algum esforço. Neste estudo a média do nível da dor, referidos pelos pacientes foi de 5 pontos, sendo considerada como dor moderada, diferentemente do estudo de da Silva et al. (2016), o qual apresentou na sua pesquisa nível de dor superior, sendo 7,1 para o grupo controle e 6,8 para o grupo de intervenção.

A origem da dor no paciente com OA tem múltiplos fatores, podendo ser ocasionada devido a rigidez articular, parestesia, má circulação na região, bloqueios articulares entre outros fatores. Observa-se que a artrose de joelho, provavelmente contribui para alterações no equilíbrio, marcha e aumento no risco de queda em pacientes idosos (Antunes et al., 2020). Isso ocorre devido ao fato de que os pacientes que apresentam osteoartrose, apresentam uma dor crônica, na qual irá repercutir consequências em diferentes fatores além da incapacidade física e funcional segundo Yamada et al. (2018).

No presente estudo, os escores da escala de equilíbrio de Berg apresentaram correlações significativas com o grau da artrose de joelho, pois, observa-se que na distribuição dos pontos segundo a EEB, metade dos pacientes tem pontuação abaixo de 36, o que corresponde, segundo Shumway-Cook et al. (2015), elevado risco de queda. No entanto, os estudos de Antunes et

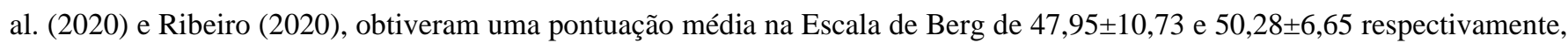
demonstrando que em média, os idosos avaliados em ambas as pesquisas apresentavam equilíbrio satisfatório com baixo risco de quedas.

Em relação ao tempo que o idoso leva para realizar o teste de TUG podemos perceber que quanto mais o equilíbrio funcional está alterado, maior é o tempo gasto para a realização da atividade física no TUG. Isso ocorre provavelmente porque com o equilíbrio alterado a insegurança do idoso é maior e desta forma promove diminuição do comprimento da passada e da velocidade da marcha, afirmou o estudo de Abdala et al. (2017). Além do o avanço da idade tornar o equilíbrio mais comprometido. O equilíbrio é um processo complexo que depende da integração da visão, da sensação vestibular e periférica, dos comandos centrais e respostas neuromusculares e, particularmente, da força muscular e do tempo de reação.

No estudo Gomes-Neto et al. (2016), os idosos avaliados apresentaram em média um tempo satisfatório no TUG, sendo 8,86 segundos entre pacientes obesos e 7,04 segundos entre pacientes não obesos. Sendo números muito próximos aos apresentados neste estudo, em que a média registrada foi de 9,8 segundos, onde $50 \%$ dos idosos fizeram as atividades em menos de 11 segundos, nos mostrando que de acordo com Gomes-Neto et al. (2016) a incidência de queda é menor dos idosos que foram avaliados nesta pesquisa.

Na avaliação do equilíbrio e da marcha através do teste de Tinetti, foi encontrado uma pontuação média de 21,4 pontos. Já no estudo de Sardim, Prado \& Pinfild (2020) também utilizando a escala de Tinetti, na primeira avaliação foi obtido uma média de 26,2 pontos para o grupo controle e 21,5 para o grupo que recebeu um tratamento com fotobiomodulação no joelho afetado, após a intervenção, novamente foi feito o teste de Tinetti e a média foi de 23,9 pontos para o grupo controle e 25,4 para o grupo da fotobiomodulação. Pode-se supor que se os indivíduos realizarem algum tratamento, esses valores podem melhorar tanto o equilíbrio quanto a marcha. 
Segundo Alves (2019) a dor crônica apresenta-se como um dos principais fatores que levam o indivíduo a sofrer com limitações funcionais, pois a dor tende a trazer adaptações ao paciente durante a execução da marcha, na tentativa de mitigar a carga sob a articulação e consequentemente a dor. Dentre as transformações estão: redução no comprimento do passo; alargamento da base de suporte; alteração no ritmo; modificação dos ângulos de flexão, extensão e adução do joelho e redução da intensidade de resposta do solo.

O presente estudo mostrou forte relação entre o nível de dor dos pacientes e a função da marcha, estando em acordo com Vargas e Silva et al. (2020), onde concluíram que a dor assim como o pensamento catastrófico da dor está fortemente relacionada com o desempenho funcional do indivíduo.

\section{Considerações Finais}

Concluiu-se nesta pesquisa, que o envelhecimento é um processo fisiológico e progressivo, no qual vários sistemas do organismo são comprometidos, principalmente o sistema musculoesquelético. Os aspectos como o equilíbrio e morbidade tem uma forte relação com o avanço da idade, podendo ter comprometimento físico e psicológico.

Devido a dor crônica que comumente é relatada pelos pacientes que apresentam artrose de joelho, suas atividades físico funcionais são comprometidas. Para tentar livrar o membro que refere a dor, o indivíduo, em determinado momento, acaba tentando criar compensações, dinâmicas ou estáticas, que podem comprometer o equilíbrio. Com isso, o indivíduo faz menor descarga de peso sobre os membros inferiores e consequentemente ocasiona alterações durante a marcha e desta forma, aumenta o risco de queda.

Porém, os dados do presente estudo não podem ser extrapolados para a população em geral, devido à baixa amostragem devido a isto, sugere-se que pesquisas com um número maior de indivíduos devam ser realizadas.

\section{Referências}

Abdala, R. P., Barbieri, W., Bueno, C. R., \& Gomes, M. M. (2017). Padrão de marcha, prevalência de quedas e medo de cair em idosas ativas e sedentárias. Revista Brasileira de Medicina do Esporte, 23, 26-30. DOI: 10.1590/1517-869220172301155494.

Alves, L. V. (2019). Correlação entre força muscular e os parâmetros espaço-temporais da marcha durante a velocidade confortável em mulheres com osteoartrite moderada de joelho.

Antunes, T. B., Silveira, T. M. V., Barbosa A. T. V., Santana, E. J., Gomes, V. M. S. A., Carvalho, V. C. P., Barros, M. L. N., Uchôa, E. P. B. L. (2020). Avaliação da marcha e do equilíbrio de pacientes idosos com osteoartrose de joelho. Brazilian Journal of Development, 6(9), 72788-72800. https://doi.org/10.34117/bjdv6n9-650

da Silva, R. M. V., Xavier, W. J. C., Neto, R. G. D., de Azevedo, V. M., do Nascimento, B. J. R., de Oliveira, J. F., \& Meyer, P. F. (2016). Efeitos da magnetoterapia no tratamento da dor na osteoartrose de joelho. ConScientiae Saúde, 15(2), 281-287. https://doi.org/10.5585/conssaude.v15n2.6245

Diegelmann, M., Schilling, O. K., \& Wahl, H. W. (2016). Feeling blue at the end of life: Trajectories of depressive symptoms from a distance-to-death perspective. Psychology and Aging, 31(7), 672-686. https://doi.org/10.1037/pag0000114

Dulay, G. S., Cooper, C., \& Dennison, E. M. (2015). Knee pain, knee injury, knee osteoarthritis \& work. Best Practice \& Research Clinical Rheumatology, 29(3), 454-461. https://doi.org/10.1016/j.berh.2015.05.005

Dutra, M. C., Cabral, A. L. L., \& Carvalho, G. de A. (2016). Tradução para o portugu6es e validação do teste Time Up Go Test. Revista Interfaces Saúde, Humanas e Tecnologia, 3(9), 81-88. https://doi.org/10.16891/2317-434X.430.ISSN

Elwyn G, Laitner S, Coulter A, Walker E, Watson P, T. R. (2017). Knee extensor strength and risk of structural, symptomatic and functional decline in knee osteoarthritis: A systematic review and meta-analysis. Arthritis Care Res (Hoboken) . (Vol. 68). https://doi.org/10.1002/acr

Ferreira, C. G., Gazzola, J. M., Cendoroglo, M. S., Dias, V. D. N., \& Ganança, F. F. (2019). Fatores associados ao equilíbrio postural de idosos longevos. Fisioterapia em Movimento, 32. https://doi.org/10.1590/1980-5918.032.AO40

Franco, M. F., Falsarella, G. R., Costallat, B. L., Coimbra, I. B., \& Coimbra, A. M. V. (2020). Associação entre osteoartrite de joelho e síndrome metabólica em pacientes idosos não institucionalizados. Revista Brasileira de Ortopedia, 55(03), 310-316. DOI: 10.1055/s-0040-1701281

Glyn-Jones, S., Palmer, A. J. R., Agricola, R., Price, A. J., Vincent, T. L., Weinans, H., \& Carr, A. J. (2015). Osteoarthritis. The Lancet, $386(9991)$, $376-387$. 
GOMES, F.R; MEJIA, D.P.M. Reabilitação de pacientes portadores de osteoartrose através da mobilização articular associados à hidrocinesioterapia Pósgraduação em Ortopedia e Traumatologia com Ênfase

Kan, H. S., Chan, P. K., Yan, C. H., Chiu, P. K. Y., Yeung, S. S., Ng, Y. L., ... \& Ho, T. (2019). Non-surgical treatment of knee osteoarthritis. Hong Kong Medical Journal. https://www.hkmj.org/system/files/hkmj_187600.pdf

Gomes, G. D. C. (2003). Tradução, adaptação transcultural e exame das propriedades de medida da Escala $i$ Performance-Oriented Mobility Assessment;(POMA) para uma amostragem de idosos brasileiros institucionalizados.

Gomes-Neto, M., Araujo, A. D., Junqueira, I. D. A., Oliveira, D., Brasileiro, A., \& Arcanjo, F. L. (2016). Estudo comparativo da capacidade funcional e qualidade de vida entre idosos com osteoartrite de joelho obesos e não obesos. Revista Brasileira de Reumatologia, 56, 126-130.

Hunter, D. J.; Bierma-Zeinstra, S. (2019). Osteoarthritis. The Lancet, 393(10182), 1745-1759. doi:10.1016/S0140-6736(19)30417-9

Hussain, S. M., Neilly, D. W., Baliga, S., Patil, S., \& Meek, R. M. D. (2016). Knee osteoarthritis: A review of management options. Scottish Medical Journal, 61(1), 7-16. https://doi.org/10.1177/0036933015619588

Kan, H. S., Chan, P. K., Chiu, K. Y., Yan, C. H., Yeung, S. S., Ng, Y. L., ... Ho, T. (2019). Non-surgical treatment of knee osteoarthritis. Hong Kong Medical Journal, 25(2), 127-133. https://doi.org/10.12809/hkmj187600

Miyamoto, S. T., Lombardi Junior, I., Berg, K. O., Ramos, L. R., \&amp; Natour, J. (2004). Brazilian version of the Berg balance scale. Brazilian Journal of Medical and Biological Research, 37(9), 1411-1421.

Monte, J. A. do ., Gomes, V. M. da S. A., Silveira, T. M. V. da, Arruda, L. Q. de, Carvalho, V. C. P. de ., Barros, M. de L. N. ., \& Uchôa, Érica P. B. L. . (2020). Influence of virtual rehabilitation with X-box ${ }^{\circledR}$ on the risk of fall s in the elderly . Research, Society and Development, 9(10), e3049108638. https://doi.org/10.33448/rsd-v9i10.8638

Nogueira, L.V., Silva, M. O., Haagen, M. V., Santos, R. C., \&amp; Rodrigues, E. L. (2017). Risco de quedas e capacidade funcional em idosos. Revista da Sociedade Brasileira de Clínica Médica, 15(2), 90-93. http://www.sbcm.org.br/ojs3/index.php/rsbcm/article/view/264.

PACCA, D. M., DE-CAMPOS, G. C., ZORZI, A. R., CHAIM, E. A., \& DE-MIRANDA, J. B. (2018). Prevalência de dor articular e osteoartrite na população obesa brasileira. ABCD. Arquivos Brasileiros de Cirurgia Digestiva (São Paulo), 31. https://doi.org/10.1590/0102-672020180001e1344

Peeler, J., Christian, M., Cooper, J., Leiter, J., \& MacDonald, P. (2015). Managing knee osteoarthritis: The effects of body weight supported physical activity on joint pain, function, and thigh muscle strength. Clinical Journal of Sport Medicine, 25(6), 518-523. https://doi.org/10.1097/JSM.0000000000000173

Rathbun, A. M., Stuart, E. A., Shardell, M., Yau, M. S., Baumgarten, M., \& Hochberg, M. C. (2018). Dynamic effects of depressive symptoms on osteoarthritis knee pain. Arthritis care \& research, 70(1), 80-88. DOI: https://doi.org/10.1002/acr.23239

Ribeiro, I. C. (2020). Relação entre gravidade radiológica e instrumentos preditores de saúde física e mental em idosos com osteoartrite de joelho.

Rodriguez-Merchan, E. C., \& De La Corte-Rodriguez, H. (2019). The role of orthoses in knee osteoarthritis. Hospital practice (1995), 47(1), 1-5. https://doi.org/10.1080/21548331.2018.1527168

Santos, João Paulo M., Andraus, Rodrigo AC, Pires-Oliveira, Deise AA, Fernandes, Marcos TP, Frâncica, Mayra C., Poli-Frederico, Regina Célia, \& Fernandes, Karen BP. (2015). Análise da funcionalidade de idosos com osteoartrite. Fisioterapia e Pesquisa, 22 (2), 161-168. https://dx.doi.org/10.590/18092950/13922922022015

Sardim, A. C., Prado, R. P., \& Pinfildi, C. E. (2020). Efeito da fotobiomodulação associada a exercícios na dor e na funcionalidade de pacientes com osteoartrite de joelho: estudo-piloto. Fisioterapia e Pesquisa, 27, 119-125.

Souza, I. F. D. S., Oliveira Neta, R. S. D., Gazzola, J. M., \& Souza, M. C. D. (2017). Idosos com osteoartrite de joelhos devem realizar avaliação nutricional: revisão integrativa da literatura. Einstein (São Paulo), 15, 226-232. https://doi.org/10.1590/s1679-45082017rw3834.

Vargas e Silva, N. C. D. O., Cardoso, T. D. S. G., Andrade, E. A. D., Battistella, L. R., \& Alfieri, F. M. (2020). Dor, incapacidade e catastrofização em indivíduos com osteoartrite do joelho. BrJP, 3, 322-327.

Yamada, E. F., Müller, F. A., Teixeira, L. P., \& Silva, M. D. D. (2018). Efeito dos exercícios de fortalecimento, de marcha e de equilíbrio no tratamento de osteoartrite de joelho. Revista Brasileira de Ciência e Movimento, 26(3), 5-13.

Zis, P., Daskalaki, A., Bountouni, I., Sykioti, P., Varrassi, G., \& Paladini, A. (2017). Depression and chronic pain in the elderly: Links and management challenges. Clinical Interventions in Aging, 12(12), 709-720. https://doi.org/10.2147/CIA.S113576. 